\title{
IRRUPCIÓN POSTFEMINISTA EN CHILE A TRAVÉS DE LAS ARTES VISUALES Y LA PERFORMANCE
}

\author{
Post-feminist eruption in chile through visual arts and performance \\ Luis Claudio Cortés Picazo* \\ María Elena Retamal Ruiz**
}

\begin{abstract}
RESUMEN
El presente artículo reflexiona críticamente en torno al carácter interrumpido que ha experimentado el feminismo en Chile como movimiento social en el transcurso del siglo XX. Heredando tareas pendientes en materia de clase, raza y género que confluyen en la problemática de la mujer latinoamericana, las cuales serán abordadas a partir de la década de los 70 por prácticas artísticas de las Artes Visuales y la Performance. Para ello se identifican y analizan ciertas operaciones artísticas que emplean el cuerpo como soporte y recurso de expresión emanadas desde los espacios marginales de creación. Concluyendo que en Chile, irrumpe un postfeminismo cuyos sujetos y agentes políticos subvierten mediante las artes visuales y performance las identidades de clase, raza y género predominantes.
\end{abstract}

Palabras clave: Feminismo, Artes Visuales, performance.

\footnotetext{
* Docente e investigador, Departamento Artes Visuales, Universidad Metropolitana de Ciencias de la Educación. Santiago, Chile. Correo electrónico: luis.cortes@umce.cl

** Docente e investigadora, Departamento Artes Visuales, Universidad Metropolitana de Ciencias de la Educación. Santiago, Chile. Correo electrónico: ivunche@gmail.com

Artículo recibido el 02 de marzo de 2016. Aceptado el 28 de agosto de 2017.
} 


\begin{abstract}
This article critically reflects interrupted around the character that has experienced feminism as a social movement in Chile during the twentieth century. Inheriting outstanding tasks on class, race and gender that shaped the problems of Latin American women, which will be addressed from the early '70s for artistic practices of Visual Arts and Performance. For it identifies and analyzes certain artistic operations that use the body as support and means of expression emanating from the marginal areas of creation. Concluding that in Chile, bursts whose subjects and politicians subvert through visual arts and performance identities of class, race and gender predominant one post-feminism.
\end{abstract}

Keywords: Feminism, Visual Arts, Performance.

\title{
INTRODUCCIÓN
}

El feminismo en Chile a lo largo del siglo XX ha presentado nudos críticos producto de interrupciones constantes en su desarrollo. Dejando tareas pendientes sin resolver sobre su base teórica y práctica como movimiento social y emancipatorio de la mujer en el contexto local. Tornándose necesario esclarecer dichos nudos ante influencias externas y entrampamientos políticos durante la primera mitad del siglo XX y posterior invisibilización frente a la irrupción violenta del golpe militar en la década del 70, junto a su tensa relación con el proceso postdictatorial (Glavic, 2015) o de transición democrática (Garretón, 2010) durante la década de los 90.

Ahora bien, en el entendido que el feminismo como movimiento social posee en origen un carácter revolucionario que vindica por los derechos de la mujer (Wollstonecraft, 1994), cabe señalar que dicha vindicación en la actualidad no atañe tan solo a las mujeres (Cortés, 2014). Hecho que ha quedado en evidencia desde márgenes institucionales a través de experiencias artísticas marginales en Chile entre la década de los 70 y los 90, mediante la irrupción postfeminista que critica la noción esencialista de mujer como sujeto y agente político del feminismo. Problemática tras la cual emerge el objetivo principal para la presente investigación, y que dice relación con reflexionar en torno a los nudos críticos que interrumpen el curso del feminismo como movimiento social en el contexto local. Contemplando por objetivos específicos el identificar y analizar aquellas estrategias empleadas por las artes visuales a través del performance que abordan dicha problemática durante el régimen militar y posterior periodo postdictatorial.

En el entendido que los nudos se presentan como instancias que permiten entrever las problemáticas de la mujer latinoamericana a partir de prácticas y operaciones artístico visuales que emplean el cuerpo y la performance como recurso de expresión. Siendo concretamente desde la marginalidad y los espacios 
de exclusión en donde el feminismo local adquiere su propio cuerpo teórico (Kirkwood, 1986).

Por lo anterior, en términos metodológicos se revisa y consulta aquella bibliografía pertinente respecto de la práctica artística local inmersa en la distinción de tres grandes bloques feministas empleados por Ana de Miguel (Amorós, 1995): feminismo pre-moderno, feminismo moderno y feminismo contemporáneo. Los cuales, ante un necesario proceso de resignificación en el contexto local, emergen los siguientes bloques feministas locales: feminismo moderno, feminismo postcolonial y feminismo "queer".

Para el primer bloque feminista es posible diagnosticar, desde el movimiento social de la mujer obrera en Chile desde el año 30, el primer rasgo performativo de reivindicación de género enmarcada en un feminismo moderno caracterizado por su asociatividad en manos del MEMCH (Montero, 2015). En el entendido que desde la acción emerge un feminismo local cuyo cuerpo colectivo de mujeres aunadas por criterios de clase, subvierten la norma imperante respecto del espacio destinado a la mujer en la sociedad y cultura a inicios del siglo XX en Chile (Luongo, 2001). De hecho el MEMCH como una organización fundada en 1935 asume la iniciativa de un grupo de mujeres de izquierda, tanto militantes de partidos como independientes, valorando la potencialidad de las mujeres dentro de la sociedad, comprendiendo que el camino de la mujer estaba en la organización y en la creación de una conciencia progresista, en el entendimiento de que no era posible una emancipación de las mujeres sin cambios radicales en todas las estructuras de la sociedad (Gaviola et al., 2007).

Para el segundo bloque feminista denominado postcolonial, cobra sentido la reflexión teórica reciente que evidencia el proceso de desmaterialización del cual fue objeto la obra de arte en el contexto de las dictaduras latinoamericanas, otorgando al cuerpo un rol subversivo a partir de la acción performática ante el blanqueamiento del cuerpo disciplinado, según Vidal (2013): espectadores encerrados en la propia obra del Grupo de Arte de Vanguardia, en Argentina ("Encierro y Escape", 1968); vocalización y sonoridad acompañada de expresiones corporales de dolor y miedo a cargo de Parra, Kay y Zurita, en Chile ("Signometraje: Tentativa Artaud" 1974); escenificación de un espacio que revive el asesinato de estudiantes a cargo del grupo Proceso Pentágono, en México (“La cámara de tortura", 1977). Procesos performáticos que según Camnitzer (2009), permitieron adentrarse en prácticas conceptuales que liberan la actividad artística de su carácter objetual, generando un comunicación estética fuera de los límites de la posición del objeto, superando el obstáculo de las fronteras geográficas.

Por último, el tercer bloque feminista denominado "queer", permite repensar en el contexto local chileno la acción performativa más allá de la práctica artística legitimada y sancionada por criterios estéticos y cánones institucionales. 
Cuya subversión central es la generada por performances artísticas ante el sancionado binarismo de género en plenos proceso de transición democrática. Generando desde la teoría "queer" (Butler, 2005) una crisis en el binarismo de género y su correlación corporal. Circunscribiendo lo "queer" en lo local bajo cuerpos de clase agresivamente erotizados, cuerpos colizas alejados de la codificación gay, cuerpos provincianos en sus gestos y desconectado de las referencias prestigiosas del arte, eclipsada con una homosexualidad marginal, lumpen y underground, según Carvajal, Delpiano y Macchiavello (2011).

\section{NUDOS CRÍTICOS DEL FEMINISMO LOCAL ABORDADOS POR LAS ARTES VISUALES Y EL PERFOMANCE EN CHILE}

Espinoza (2009) nos señala que en el contexto latinoamericano la producción de una reflexión sobre la identidad y sobre los cuerpos del feminismo se ha desarrollado en base a marcos conceptuales importados, sin que mediaran intentos de reapropiación que permitieran aterrizar ese cuerpo en la materialidad de los cuerpos racializados, empobrecidos, folclorizados, colonizados de las mujeres latinoamericanas. Por ende, figura de manera relevante las aportaciones otorgadas por las artes visuales desde el performance como género artístico a partir de la década de los 70 . Especialmente las acciones de artes centradas en la realización escénica teniendo como eje central el cuerpo del o la artista. Diferenciándolo del teatro por cuanto el performance no es representación, sino más bien presentación del cuerpo sobre una estructura narrativa desprovista de ficción y simulacro (Fischer-Litcher, 2013). Subvirtiendo de este modo los lenguajes disciplinares de creación visual, debido a su carácter híbrido y polimorfo, transformando la cultura circundante a través de la acción del cuerpo del artista mediante la articulación de lenguajes visuales, corporales gestuales, verbales y escénicos (Ferrer, 2014).

A continuación se identifica y analiza el desarrollo del feminismo en Chile en el transcurso del siglo XX, para efectos de comprender su dinámica interrumpida que deja tareas pendientes en materia de clase, raza y género. Las que serán atendidas críticamente desde las artes visuales y acciones artísticas que encuentran en la performance una estrategia clave para subvertir la relación política entre feminismo y mujer. Para dicho efecto se emplea una estructura de comprensión del feminismo basado en tres bloques: Feminismo moderno, Feminismo Postcolonial y Feminismo "queer".

\section{Feminismo Moderno}

Debido a que no es posible detectar el feminismo pre-moderno en Chile en los términos señalados por Ana de Miguel (Amorós, 1995), cabe destacar que 
iniciado el siglo XX se dan las primeras luces de un feminismo de carácter moderno. Antecediendo de este modo el primer nudo que más adelante haremos referencia.

De hecho antes de hablar en términos modernos de movimiento feminista en Chile según Gaviola, Jiles, Lopresti y Rojas (2007), se debe hablar primeramente del despertar de la conciencia feminista. Esta conciencia que entreteje varios factores; algunos como la dictación del decreto Amunátegui en 1877 (Eltit, 1994) que permitió a las mujeres acceder a la enseñanza superior, junto a su incorporación al trabajo asalariado, propiciando lentamente el ascenso del movimiento feminista con una sólida conciencia no menos política.

Concretamente a partir de 1910 el feminismo gestado a nivel local adquiere luces de modernidad tras los siguientes factores: la constante influencia extranjera, la lucha por el derecho a voto, derechos civiles equivalentes a los del hombre, dentro y fuera del matrimonio y, preocupaciones ajenas a la realidad local, tales como los dos conflictos bélicos internacionales; acompańado evidentemente de la cuota solidaria que caracterizará la oleada organizativa para atender la igualdad de derechos sociales, civiles y políticos de la mujer en Chile (Gaviola et al., 2007). Siendo uno de sus aspectos de mayor impacto, la emergencia de la instalación del feminismo bajo la tensa relación entre clases sociales con marcado acento político. Derivando en una conciencia feminista acompañada de una clara distinción de clase derivada de dos tendencias políticas: la conservadora atribuida a mujeres simpatizantes con la Iglesia católica (Maza, 1995) y la de izquierda simpatizante activa del Partido Comunista y en especial del Frente Popular (Gaviola et al., 2007). Sanción y subversión que sitúa a las mujeres en Chile ante un feminismo tensionado por la variable de clase. Predominando un fuerte discurso amoroso en los círculos de élites (Luongo, 2001), mientras que la mujer obrera proveniente de la explotación de salitre se organiza sindicalmente para velar por la integridad de sus familias, y muy particularmente sus hijos e hijas (Montero, 2015).

Asentandolas bases de un feminismo moderno decarácter organizado yasociativo con clara tendencia de izquierda bajo dos organizaciones. La primera denominada Movimiento Pro Emancipación de la Mujer Chilena (MEMCH) en 1935, y la segunda denominada Federación Chilena de Instituciones Femeninas (FECHIF) en 1946. Dando luces modernistas al movimiento desde la FECHIF al estar presidida en sus inicios por Amanda Labarca (1886-1975), cuyo texto "Feminismo Contemporáneo" de 1947 lo preconizaba. Bajo este contexto emerge en Chile un feminismo moderno centrado principalmente en la obtención del derecho a voto para las mujeres, el cual será alcanzado en 1949. Feminismo moderno que poseerá características importadas desde feminismo sufragista, a pesar de su carácter tardío en comparación al resto de los países europeos protestantes y católicos (Maza, 1995).

Sin embargo, la obtención del derecho a voto femenino no estará exenta de polémica, ya que beneficiará principalmente los intereses de mujeres de elite y clase 
conservadora pertenecientes a la Asociación de Dueñas de Casa de 1947 liderada por Rosa Markmann, esposa del presidente Gabriel González Videla. Lo que trajo consigo la ilegalización del Partido Comunista. Generando de este modo el primer nudo en el pensamiento del movimiento feminista local. Despojando al feminismo de su carácter asociativo y organizativo tras la ilegalización del Partido Comunista al cual se encontraban adscritas mayoritariamente mujeres de sectores populares (Gaviola et al., 2007). Dejando pendientes la liberación de la mujer en igualdad de condiciones al hombre, ante la sujeción de un marco cultural androcéntrico de clase.

\section{Feminismo Postcolonial}

Se comprende por feminismo Postcolonial, al feminismo enmarcado en la Tercera Ola (Gimeno, 2009) por cuanto se centra en la diversidad de las mujeres. Cuyo carácter anti-esencialista atiende las diferencias entre las mujeres sin reproducir las nociones esencialistas de las diferencias culturales, basándose e insistiendo en la diferencia (Amorós, 1995). Dicho concepto utilizado por primera vez en 1980 conserva una estrecha relación con los planteamientos del feminismo posmoderno y postestructuralista, debido a sus características asentadas en las diferencias, flujos, mixturas, identidades alternativas y múltiples. Por cuanto reflexiona desde la cotidianeidad y subjetividad de casos particulares (Oliva, 2004).

Ahora bien, considerando las tareas pendientes del feminismo moderno tras la obtención del sufragio de la mujer en Chile, emerge una Tercera Ola, sin atender necesariamente del todo una Segunda Ola. Identificándose un segundo nudo por cuanto en el marco de la cultura occidental la Primera Ola del feminismo luchó por el sufragio y acceso a la educación, mientras la Segunda Ola atendió el acceso igualitario al espacio público, emergiendo repentinamente en Chile una Tercera Ola que se identificará con la desafección de las jóvenes por el feminismo tradicional.

La Tercera Ola feminista en Chile en la década los 70 emerge a raíz de la irrupción violenta del Golpe Militar. Gestándose fuertemente dos visiones feministas: multiculturalista (Amorós, 2004) y "queer" (Butler, 2005; Preciado, 2002).

Respecto del Feminismo Multicultural según Amorós (Cobos, 2005), se abre la posibilidad de visibilizar un proceso ilustrado a nivel local, al margen de los modelos importados desde Europa y Occidente, puesto que no tendrían el monopolio de la Ilustración y por ende de un feminismo universal: "Si existe una relación intrínseca entre feminismo e Ilustración y resulta que puede haber varias Ilustraciones, debemos concluir que hay feminismo en plural" (Cobos, 2005, p. 115). Abriendo la posibilidad de seleccionar criterios pertinentes para la elaboración de un feminismo multicultural relacionado al contexto local. 
Feminismo Multicultural de carácter local alimentado especialmente por de María Lugones, quien bajo una mirada del feminismo de raíz latinoamericana atiende la fragmentación de la noción de sujeto universal. De hecho la autora, según Oliva (2004), distingue entre multiplicidad (mestizaje), fragmentación, unificación y homogeneidad como principios relacionados con la ordenación del mundo social: "El yo mestizo... desemboca en el mestizaje en el mundo social; en cambio, la fragmentación dentro de los individuos está conectada con la orientación de lo múltiple proyectado hacia un mundo social homogéneo" (Oliva, 2004, p. 11). Problemáticas que por lo demás serán abordadas en Chile durante la dictadura militar bajo dos variables emergentes en el mundo posmoderno, tales como clase y etnicidad.

Así por ejemplo, bajo este contexto en Chile, Kirkwood (1986) canaliza las reivindicaciones ante el orden predominante de lo masculino en la sociedad chilena, no tan solo el interior del hogar, sino además, fuera de él. Mientras que Montecino (2003) nos aproxima críticamente a los íconos sagrados universales representativos del culto hacia el Dios Padre eurocéntrico e irrupción violenta de las fuerzas armadas al interior del espacio doméstico feminizado, producto del proceso de mestizaje heredado desde los tiempos de conquista.

Por último, respecto del feminismo "queer", Oliva (2004) nos aproxima ante el tratamiento disciplinado del cuerpo social de la sociedad chilena hasta su más íntima corporeidad, coincidente con el feminismo lesbiano y/o negro que se despegan del feminismo de vertiente tradicional de la década de los setenta, dando lugar a un tipo de feminismo que dirige sus críticas al racismo y al etnocentrismo. Desidentificándose con las formulaciones predominantes del sujeto teórico del feminismo igualitario basado en la formulación sólida del concepto de mujer. Emergiendo fuertemente el concepto "queer" por cuanto reivindica las identidades múltiples y deconstruye los pares binarios de sexo y género, que mantienen inmutable el concepto de mujer como sujeto y agente político del feminismo.

Argumentaciones teóricas que enmarcan operaciones artísticas locales de las artes visuales y el performance, entorno a la transgresión de las fronteras de género tras la confluencia Arte y Política mediante la Escena de Avanzada y el Colectivo de Acciones de Artes C.A.D.A en Chile. Destacando la presencia de los/las siguientes artistas: Carlos Leppe (1975), Lotty Rosenfeld (1979), Marcela Serrano (1979, 1980), Diamela Eltit (1980), Diamela Eltit y Lotty Rosenfeld (CADA, 1982) y Paulina Humeres (1982). Quienes aluden la necesidad de desacatar la instauración e irrupción violenta de un modelo político sin previo consenso ciudadano mediante la experiencia artística performativa al margen de las instituciones.

Así por ejemplo Carlos Leppe con su obra "El perchero" de 1975 anula su género e identidad sexual, operando su cuerpo como objeto frente al cuerposocial, transcurriendo entre lo íntimo y privado de su experiencia corporal en 
cuanto al ocultamiento de su ser entablando una relación contingente con los acontecimientos de intervención violenta de los cuerpos en términos de género (femenino/masculino) por parte de la dictadura militar:

...entre su cuerpo biográfico y el cuerpo social histórico, entre su cuerpo como ser sexuado y los otros cuerpos sexuados...su cuerpo como matriz, como soporte desnudo, fue interferido y bloqueado con vestidos, vendas y gasas, que cubrieron y disimularon su identidad sexual y simularon al mismo tiempo, un acto de castración (Ivelic y Galaz, 1988, p. 197).

Lotty Rosenfeld en su obra "Una milla de cruces sobre el pavimento" de 1979, en Santiago de Chile, pone a prueba la relación transgresora entre cuerpo, signos y poder de una ciudad militarizada como señal de desacato, acto de transgresión de un subsistema de tránsito en un país enteramente regimentado que va mucho más allá de lo denotado por el pavimento.

Marcela Serrano en 1979 experimenta su cuerpo como soporte u obra (corps-trouve) estrechando un abismo entre las acciones rituales con contenido mágico-mítico y, la experiencia de intervención violenta del cuerpo ciudadano en plena dictadura militar. En 1980 con "Cuadro Clínico" realiza un trabajo analógico atendiendo el caso de Dora mediante un análisis fragmentario sobre la histeria de 1924 realizado por Freud. Para lo cual emplea su propio cuerpo como mecanismo de desciframiento de la histeria, adoptando poses cliché frente al lente fotográfico, emulando el tratamiento del género femenino como objeto de consumo por los medios de comunicación, aproximándose de este modo a un diagnóstico o cuadro clínico realizado con clara distinción biológica, abordando la problemática de la representación de la mujer chilena en estereotipos culturales con clara base sexista durante la dictadura militar (CADA, 1982).

Bajo este contexto, Diamela Eltit a partir de la década de los 80 transforma su cuerpo convirtiéndolo en cuerpo sacrificial, interpelando el cuerpo sexuado de la mujer al asumir la culpa y el dolor colectivos transitando por prostíbulos, cárceles y hospicios designados como "Zonas de dolor" (1980): "En esta obra, la autora, precisamente sitúa su cuerpo, su imagen y su palabra, mientras lava la calle, purificándola de un acto simbólico de penitencia y arrepentimiento colectivos" (Ivelic y Galaz, 1988, p. 217).

Es así como Eltit y Rosenfeld con motivo de la conmemoración de El Día Internacional de la Mujer, invitan en nombre del Círculo de la Mujer, a participar de la exhibición de un film pornográfico denominado "Un Filme Subterráneo" en 1982. El cual es protagonizado por un triángulo configurado por una patrona, una sirvienta y un perro. Dicho filme, realizado en Súper 8, recibe la denominación de subterráneo al permanecer fuera de los circuitos comerciales de distribución, 
ya sea por su contenido censurado o por su exacerbación y contenido erótico. Escenificando las supuestas fantasías sexuales que, en la cotidianeidad, permanecen erráticas y que ubican al espectador masculino en la clasificación psicopatológica de voyerista (CADA, 1982).

Por último, Paulina Humeres quien mediante una performance elabora su obra EROICA de 1982, en donde la artista se presenta desnuda en una sala y sobre su cuerpo se proyecta una diapositiva con la imagen de Eva, pintada por Palma IL Vecchio. Posteriormente la artista se pinta el cuerpo de color rosado y se imprime sobre 10 pliegos de papeles montados sobre el muro donde ya existe un dibujo previo de su silueta. La acción además de ser fotografiada es grabada en video y simultáneamente proyectada en monitores. Luego de la impresión la artista pinta con amarillo limón el fondo de la pintura y escribe con espray negro la palabra EROICA.

\section{Feminismo "queer"}

Una vez finalizado el régimen militar, durante la primera década del periodo postdictatorial se identifica un tercer nudo en la agenda feminista. El cual, será abordado por prácticas artísticas de las artes visuales y el performance desde espacios marginales. Atendiendo tareas pendientes tales como la igualdad entre los géneros, la visibilización y legitimación de los grupos minoritarios de sexo y género en Chile correspondientes a la Tercera Ola.

Tensionando relaciones con la oficialidad basada en acuerdos y pactos políticos de clase, ante la emergente reflexión feminista proveniente desde espacios de exclusión, tales como lo "queer". Término cargado de un estigma paralizante de interpelación mundana de una sexualidad patologizada, el usuario del término transforma el término en emblema y vehículo de la normalización y regulación discursiva de los límites de la legitimidad sexual (Butler, 2005).

Dicho tercer nudo impidió la discusión feminista desmarcada de las políticas de género y Planes de Igualdad. Oficializando la noción esencialista de mujer como sujeto y agente político del feminismo institucional bajo el alero del Servicio Nacional de la Mujer (SERNAM). Evidenciándose claras debilidades ante el respeto por los derechos humanos en pleno proceso de retorno a la democracia ante la no aceptación de las nuevas identidades de género LGBTI en Chile a nivel legal.

Garretón (2010) en este sentido menciona que una vez finalizado el régimen militar en Chile, el proceso de transición hacia la democracia (la modernidad como proyecto inacabado) manifiesta contradicciones y debilidades en su dimensión ciudadana: "problemas de derechos civiles específicos de las minorías, desigualdades socioeconómicas, participación electoral y ausencia de instituciones de participación” (Garretón, 2010, p. 116). 
Bajo estos términos se presenta como tarea pendiente la deconstrucción de la identidad de género que predomina bajo el dispositivo sexo-género (Rubin, 2008), por cuanto la ACNUDH (2015) señala que los Estado Parte (en el Pacto Internacional de Derechos Económicos, Sociales y Culturales) deberían asegurar que la orientación sexual de una persona no sea una barrera para alcanzar los derechos del Pacto. Tras lo cual se desprende que Chile contempla tareas pendientes en materia de grupos minoritarios LGTBI, según la Organización de Transexuales por la Dignidad de la Diversidad (OTD, 2012):

Los grupos de mujeres transexuales e intersex, deben lidiar con aquella parte de la sociedad chilena que considera válido el machismo y la misoginia, que discrimina, hostiga, persigue y está dispuesta a llegar a asesinar a un ser humano sólo por ser diferente (49).

Tales antecedentes reflejan que durante la primera década del periodo postdictatorial, Chile no reflexiona desde la oficialidad en torno al feminismo local al margen del Servicio Nacional de la Mujer o SERNAM. Transitando bajo el siguiente mandato: "desde el primer gobierno de la Concertación hasta hoy a hacer operativos los mandatos de género más bien conservadores y controladores de la (in)diferencia sexual" (Olea, 1999, p. 58).

Siendo que el feminismo tras su instancia poscolonial de los 80 surca por diversas modificaciones en su vertiente "queer", el cual postula teóricamente repensar el objeto de su acción política y reivindicaciones del movimiento. En el entendido que desde lo "queer" se argumenta la necesidad de deshacer el género y adentrarse en la crítica feminista "queer", cuyo cuestionamiento implica perder el sentido que otorgamos el ocupar un género. Generando una crisis en la ontología experimentada a nivel de la sexualidad y el lenguaje, ante el surgimiento de nuevas formas de pensar el género a la luz de lo transgénero y la transexualidad, la paternidad y maternidad lésbicas y gais, y las nuevas identidades lésbicas masculinas y femeninas (Butler, 2005).

Por ende, cabe que reflexionar críticamente ante las nuevas identidades de género en Chile, con clara y sustentable base teórica "queer". Debiendo abordar desde el feminismo el derrocamiento y supresión del género, convirtiéndolo en algo ambiguo: "precisamente porque siempre es un signo de subordinación de la mujer" (Butler, 2007, p. 15). Es así como, el feminismo made in occident (Amorós, en Cobos, 2005) ha de ser derrocado con el objeto de dar lugar al cuestionamiento de la universalidad como forma de exclusividad negativa y excluyente (Butler, 2007). En donde la identidad de género unívoca, bajo su relación sexo-género conforma una noción errónea de identidad.

Es así como desde las prácticas artísticas marginales a raíz del régimen militar en Chile, el tratamiento del cuerpo generizado en un marco de representación y 
disciplinamiento del cuerpo social y doméstico, heredan al periodo postdictatorial una tarea pendiente. La que dice relación con la discusión del feminismo desaprendido de las categorías de mujer y por ende de géneros binarios por oposición. Tal es el caso, por ejemplo, de las propuestas y operaciones artísticas experimentales presentes en las obras de Las Yeguas del Apocalipsis (1989, 1990) y Juan Domingo Dávila (1946-).

Experiencias las cuales abren la discusión Postfeministas en el espacio local de raigambre postcolonial, desde donde Las Yeguas del Apocalipsis (1989, 1990) enfrentan radicalmente la heterosexualidad imperante en un país machista, empleando una relación interdisciplinar entre escritura y creación artística visual. Y Juan Domingo Dávila (1946-) quien durante la transición postdictatorial con sus obras Cita Amorosa (1985), El libertador Simón Bolivar (1994) y La Rota (1996), desestructura el soporte tradicional pictórico empleando un lenguaje de proyección del "Yo" psicológico, configurando una propuesta visual autobiográfica basada en el cruce de fronteras de sexo-género.

Durante los setenta y ochenta, en plena dictadura la obra de Juan Dávila y Carlos Leppe conceptualizan arte y homosexualidad (dentro de un espacio restringido por la propia cultura de oposición a la dictadura). Siendo Dávila de la postdictadura y Las Yeguas del Apocalipsis quienes enlazarán la categoría del travestismo enmarcada en lo "queer". De hecho, según Carvajal (Carvajal, Delpiano y Macchiavello, 2011):

...desde fines de los años '70 el medio cultural chileno vio irrumpir, de la mano de Juan Dávila y Carlos Leppe, un conjunto de prácticas que subvertían las concepciones binominales de género, dichas prácticas no tuvieron anclaje a la letra de una teoría homosexual (20).

Por ende cabe señalar, en primer lugar, que Pedro Lemebel y Francisco Casas como integrantes de Las Yeguas del Apocalipsis, emplean como recurso de expresión, la elocuencia del propio cuerpo abyecto, así como el cuerpo de otros, desaparecidos o torturados (Mosquera, 2006). Seńalando que a través del travestismo, la estética de lo pobre y kistch, se lanza una protesta contra la dictadura, y además contra de la democracia que se pactaba a fines de los 80 , que los dejaba igualmente marginados. En este sentido, Robino (1991) nos menciona que desafiaron el duelo con miradas oblicuas causando placeres y arcadas, identificándose con el "lumpen”, es decir, con el último desecho de una sociedad de consumo, el que recicla toda la información del glamour de las divas de Hollywood.

Dentro de su producción artística, cabe destacar el registro visual de una performance desarrollada en la Facultad de Artes de la Universidad de Chile en 1989, institución que por lo demás, se encontraba intervenida por los militares. De 
este modo, los autores, desnudos y montados a caballo, acceden a dicha institución simbolizando metafóricamente la refundación de la Universidad junto al ingreso de las minorías en la academia. Salas (1989, p. 27), al respecto menciona lo siguiente: "Montados a caballo, en pelotas, yegua sobre yegua o una yegua de dos pisos, refundando la Universidad de Chile, en las Encinas".

Posteriormente los/las autores/as realizan una performance-instalación en la Galería Bucci en 1990 en donde el cuerpo de ambos/as se personifica y trasviste con el objeto de citar una obra clave de Frida Kahlo (1907-1954). Para dicho efecto se proyecta una foto del cuadro homónimo de Frida Kahlo desde la calle hacia el interior de la galería, haciendo que la imagen se difuminara al traspasar un plástico colgado en la entrada y otro en el interior. Yaciendo en el suelo la foto escenificada de dicho cuadro que los/las artistas representaron al fondo de la galería, con sonda de transfusión, sangre y pintura al óleo sobre el cuerpo.

La obra pone en cuestión la categoría mujer y hombre como identidades estables y polarizadas, cuya copia en vivo de la pintura de Khalo, critica el patetismo que circunda el mito de Khalo como mujer-víctima. Espectáculo de una contorsión homosexual montada desde el arte de la performance, según Richard (2001):

La oblicuidad femenizante del travestismo iba destinada a perturbar el control de una verdad-del-saber, con sus enredos cosméticos y sus intrigas simulacionales...la obra descolocaba el mercado de las representaciones de identidad con su parodia travesti que carnavaliza tanto lo femenino como la iconización feminista de Frida Khalo (p. 212).

Por último, Juan Dávila aborda críticamente el elitismo cultural y aristocrático del arte en Chile, desestructurando el soporte tradicional pictórico empleando un lenguaje de proyección del Yo psicológico que le permite configurar una propuesta visual autobiográfica. Richard (1985), en relación a Cita Amorosa de Juan Dávila, señala que el artista elabora un discurso en torno a su experiencia de viaje, como etapa que marca discontinuidad en la rutina. Tanto así, que lo lleva al plano de traspasar fronteras no tan solo geográficas (Chile-Australia), sino de orden simbólico-cultural, entre lo masculino y lo femenino (travestido). Parte de la experiencia del Yo en proceso de conformación, no dentro de una continuidad que lo acepte tal cual como lo dictan las normativas genéricas, sino más bien, como un travestido, mutante, cambiante y discontinuo (Richard, 1985).

Con su obra, Dávila otorga múltiples lecturas y sentidos al ámbito de lo obsceno; cuya comprensión como experiencia irregular, perturba al espectador con su brutal y violenta recreación pictórica de escenas pornográficas provenientes del cine y fotografía mientras subvierte toda educación visual de aquello que ha permanecido oculto dentro de las reglas de lo inmostrable. 
La recreación de fragmentos corporales sexuados en la pintura de Dávila no es casual. Históricamente, la pintura occidental ha construido cuerpos sexuados ideales. Dávila los cuestiona. Y, al menos, el cuerpo femenino es objeto de su travestismo al despojarlo de toda idealización materna e inmaterialidad otorgada por la tradición pictórica. Por ello, al emplear la imagen pornográfica, el desnudo femenino es encuadrado, fragmentado, exhibido en recortes, empleando recuadros y close-up. Por ejemplo, en Cita Amorosa es un proxeneta de la pintura: “...favorece relaciones ilegítimas -y contaminantes- entre las diferentes técnicas de producción y con consumo visual que rivalizan en la historia" (Richard, 1985).

En plena década de los 90 integra de la denominada Escuela de Santiago (Richard, 2001), a partir de allí pone en circulación una tarjeta postal sobre la reproducción de una imagen de Simón Bolívar (1783-1830) travesti, financiada por el Ministerio de Educación. La obra polemizó en torno a la libertad de creación o censura artística, discriminación homosexual, respeto por las diferencias, pluralismo ideológico y modernidad cultural. En dicha obra se planteaba una América Latina como una construcción mestiza no solo en términos del cruce étnico, sino también de las hibridaciones de códigos que entrechocadamente unen modernidad y tradiciones, vanguardia y folclor, estéticas metropolitanas y culturas populares, conjugando paródicamente lo masculino y lo femenino en una zona de ambigüedades sexuales que lleva la marca de la indefinición, el tumulto de incerteza (Richard, 2001).

En esta línea, su obra La Rota de 1996 al citar la obra La perla del Mercader de Alfredo Valenzuela Puelma (1856-1909) manifiesta que el personaje de El roto posa en lugar de la esclava que va a remate público, esperando la mano alzada del mejor postor, del único postor. Así, el roto desplaza la imagen clásica de la esclava y se erige en un femenino por cuya carne transita un otro no menos esclavista, en una estrategia que articula un discurso crítico que plantea, en primer término, un proceso de desublimación de la pintura mediante la tematización de relaciones sexuales aberrantes, donde el travestismo, mediante el disfraz y el maquillaje, altera violentamente la identidad sexual de los personajes propuestos por el pintor (Eltit, 1996).

\section{CONCLUSIÓN}

Se concluye tras identificar los tres nudos críticos del feminismo local, que durante su desarrollo en el siglo XX el análisis de las tensiones de clase, raza e identidades de género se efectúa a partir de operaciones artísticas de las artes visuales y perfomance. Operaciones las cuales deben lidiar ante el predominio de dos mecanismos oficiales asentados culturalmente durante las tres últimas décadas del siglo XX. Primero, el mecanismo del terror instalado sin consenso tras el golpe 
militar. Segundo, el mecanismo de mercado producto de acuerdos excluyentes durante la postdictadura (Rojas, 2012).

Respecto de su análisis cabe señalar que en términos de clase, el feminismo moderno dejará una tarea pendiente por resolver por cuanto los aspectos revolucionarios que vindicaba por los derechos de la mujer chilena se verán entrampados ante decisiones políticas que privilegió a las mujeres de clases acomodadas. En términos de raza, la tarea pendiente es producto de la irrupción violenta y armada del cuerpo social íntimo y público en Chile que instauró un blanqueamiento que subsumió el trauma del proceso de mestizaje del cual la mujer fue objeto. En términos de identidades de género, la tarea pendiente será con los cuerpos minoritarios cuya representatividad quedará subsumida por los pactos y acuerdos de postdictadura enmarcada por la heteronormatividad.

Por lo tanto, las artes visuales mediante estrategias y operaciones no disciplinares, subvierten el lenguaje de creación visual empleando el cuerpo y performance para atender las problemáticas de clase, raza y género para su reflexión crítica. Reflexión que efectuada desde los espacios comunes de exclusión y marginación que cada obra aborda desde la práctica de su propio/a autor/a. Cobrando sentido repensar el feminismo como movimiento revolucionario y emancipatorio, no tan solo desde la mujer, sino además desde los cuerpos sin sexo y sin género, cuyas problemáticas de clase y raza han sido atendidas críticamente desde la acciones artística disruptivas, en la medida que sus creadores/as palpan sobre su propio cuerpo y experiencia la exclusión de las cuales han sido objeto.

\section{REFERENCIAS}

ACNUDH, "Combatting discrimination based on sexual orientation and gender identity", Naciones Unidas Derechos Humanos. Oficina del Alto Comisionado para los Derechos Humanos. Disponible en: http://www. ohchr.org/EN/Issues/Discrimination/Pages/LGBT.aspx. [Consultado el 10 de junio de 2015].

Amorós, Celia. "Por una ilustración multicultural", Quaderns de filosofía i ciencia 34, (2004): 67-79.

Amorós, Celia. Diez palabras claves sobre mujer. Navarra: Verbo Divino, 1995.

Butler, Judith. Cuerpos que importan. Sobre los limites materiales y discursivos del sexo. Buenos Aires: Paidós, 2005. 
Butler, Judith. El género en disputa. El feminismo y la subversión de la identidad. México: Paidós, 2007.

C.A.D.A. Ruptura: Documento de arte. Santiago de Chile: Ediciones C.A.D.A, 1982.

Camnitzer, Luis. De la Coca-Cola al Arte Boludo. Metales Pesados. Santiago de Chile, 2009.

Carvajal, Fernanda, Delpiano, María José y Macchiavello, Carla. Ensayos sobre Artes Visuales. Prácticas y discursos de los años '70 y '80 en Chile. LOM. Santiago de Chile, 2011.

Cobos, Rosa. "El género en las ciencias sociales", Cuadernos de Trabajo Social 18 (2005): 249-258.

Cortés, Luis. "La cultura visual como contenido vital de la educación artística en Chile: las heroínas como recurso didáctico del siglo XXI". Tesis doctoral. Espańa: Universidad de Complutense de Madrid, 2014.

Eltit, Diamela. "Quehacer crítico y disidencia", La Época 273 (1989): 5.

Eltit, Diamela. Crónica del sufragio femenino en Chile. Santiago de Chile: SERNAM, 1994.

Eltit, Diamela. La rota. Juan Dávila. Santiago de Chile: Galería Gabriela Mistral, 1996.

EROICA. "Chile años 70 y 80. Memoria y Experimentalidad", Santiago de Chile, Cuadernos Museo de Arte Contemporáneo, Facultad de Artes, Universidad de Chile (1982): 14-15.

Espinoza, Yuderkis. "Etnocentrismoy Colonialidad en los Feminismos Latinoamericanos", Revista Venezolana de Estudios de la Mujer 14/33 (2009): 37-54.

Ferrer, Esther. "Esther Ferrer: Que me den un premio no me van a callar la boca". Disponible en:http://www.abc.es/cultura/arte/20141113/abci-esther-ferrerpremio-velazquez 201411121817.html. [Consultado el 10 de junio de 2015]. 
Fischer-Litcher, Erika. Estética de lo Performativo. ABADA Editores: Madrid, 2013.

Garretón, Manuel Antonio. "La Democracia Incompleta en Chile: la realidad tras los Rankings Internacionales”, Revisa de Ciencia Politica 30/1 (2010): 115-148.

Gaviola, Edda, Jiles, Ximena, Lopresti, Lorella y Rojas, Claudia. Queremos votar en las próximas elecciones. Historia del movimiento sufragista chileno 19131952. Santiago de Chile: LOM, 2007.

Gimeno, Beatriz. ¿Qué feminismo? Disponible en: http://www.trasversales.net/ t16bg.html. [Consultado el 30 de noviembre de 2016].

Glavic, Karen. "Diálogos filosóficos: Memoria y Postdictadura: voces femeninas de la filosofía en Chile". Disponible en: http://www.uchile.cl/multimedia/112025/ dialogos-filosoficos-memoria-ypostdictadura-voces-femeninas. [Consultado el 03 de junio de 2015].

Ivelic, Milán y Galaz, Gaspar. Chile arte actual. Valparaíso. Valparaíso de Chile: Ediciones Universitarias de Valparaíso y Universidad Católica de Valparaíso, 1988.

Kirkwood, Julieta. Ser Politica en Chile. Las Feministas y los Partidos. Santiago de Chile: FLACSO, 1986.

Luongo M., Gilda. "Acción feminista y contradicción en la discursividad de Amanda Labarca”, Universum 16 (2001):143-151.

Maza, Erika. "Catolicismo, anticlericalismo y la extensión al sufragio a la mujer en Chile”, Estudios Públicos 58 (1995): 137-195.

Montecino, Sonia. Madres y Huachos: Alegorías del mestizaje chileno. Santiago de Chile: Editorial Sudamericana, 2003.

Montero, Claudia. "La conformación de discurso feminista en diálogo con los discursos sociales: las mujeres frente a los problemas sociales del 30", Universum 30 (1) (2015): 153-171.

Mosquera, Gerardo. Copiar el edén. Arte reciente en Chile. Santiago de Chile: Puro Chile, 2006. 
Olea, Raquel. "Femenino y feminismo en transición: Transición: ¿Un debate Abierto?", Revista Encuentro XXI 15 (1999): 56-62.

Oliva, Asunción. "Feminismo Postcolonial: La crítica al eurocentrismo del feminismo occidental”. Disponible en: http://www.museo-etnografico.com/ pdf/puntodefuga/160120oliva.pdf. [Consultado el 06 de marzo de 2011].

Preciado, Beatriz. Manifiesto contra-sexual. Madrid: Opera Prima, 2002.

Proyecto de Ley sobre Identidad de Género que reconoce y da protección al derecho a la identidad de género. Disponible en: http://bibliotecadigital. indh.cl/handle/123456789/648. [Consultado el 10 de junio de 2015].

Richard, Nelly. "La problemática del feminismo en los años de la transición en Chile", Estudios Latinoamericanos sobre cultura y transformaciones sociales en tiempos de globalización. Buenos Aires, CLACSO (2001): 227-239.

Richard, Nelly. Arte en Chile 1973. Escena de Avanzada y Sociedad. Santiago de Chile: FLACSO, 1987.

Richard, Nelly. La Cita Amorosa (Sobre la pintura de Juan Dávila). Santiago de Chile: Francisco Zegers Editor, 1985.

Robino, Carolina. "Las últimas locas del fin del mundo: Las Yeguas del Apocalipsis", Hoy 736 (1991): 42-45.

Rojas, Gonzalo. Las obras y sus relatos II. Santiago de Chile: Departamento de Artes Visuales Universidad de Chile, 2012.

Rubin, Gayle. "Continuidad y cambio. Género y culturas de la tecnología en el trabajo”, Telos. Cuadernos de comunicación e innovación 74 (2008): 48-55.

Salas, Fabio. "Casas Las yeguas del Apocalipsis”, Cauce 204 (1989): 26-29.

Vidal, Sebastián. "El espacio y el cuerpo de la tortura: algunos casos de su representación artística en Latinoamérica”. Disponible en: http://arteycritica.org/autores/ sebastian-vidal-valenzuela/ [Consultado el 30 de noviembre de 2016].

Wollstonecraft, Mary. Vindicación de los derechos de la mujer. Madrid: Cátedra, 1994. 